\title{
¿Por qué numerus clausus en medicina? Razones para frenar la apertura de nuevas facultades de medicina
}

Foro de la Profesión Médica de España

- El buen funcionamiento del Sistema Nacional de Salud (SNS) se asienta en la calidad de la formación de los profesionales sanitarios.

- Garantizar la calidad de la formación en las facultades de medicina merece una atención muy especial.

- Con el título universitario de grado, los médicos no están habilitados para el ejercicio asistencial, sólo son 'médicos nominales.'

- Medicina es la carrera universitaria más larga. Para ejercer, son necesarios 11 años de formación universitaria y especializada.

- Las plazas MIR convocadas han disminuido en las dos últimas convocatorias un 10\% y los estudiantes que acceden a estudiar medicina, sólo un $1,35 \%$.

- El desajuste entre la oferta de plazas MIR y los egresados de las facultades de medicina será de más de 1.000 médicos anuales.

- A finales de 2013 había 2.389 médicos en paro, 4.203 eran demandantes de empleo y, a lo largo del año, emigraron 3.400.

- En ocho años, se ha pasado de 28 a 40 facultades $y$ de 3.450 a 7.000 estudiantes que acceden a las facultades de medicina.

- Actualmente, España es el segundo país de más de 20 millones de habitantes en número de facultades de medicina y, si no frenamos la apertura de nuevas facultades, podríamos alcanzar el primer puesto.

- No es congruente reducir el número de estudiantes de medicina y abrir nuevas facultades de medicina.

El Foro de la Profesión Médica de España, que agrupa a las organizaciones más representativas de los médicos, ha alzado su voz al unísono en defensa de la calidad de la formación de los profesionales, en la que se asienta el buen funcionamiento del SNS, y para poner freno a la apertura de nuevas facultades de medicina y adecuar el numerus clausus a las ne- cesidades reales de las plazas MIR en los hospitales acreditados.

La Organización Médica Colegial de España (OMC), la Confederación Estatal de Sindicatos Médicos (CESM), la Federación de Asociaciones Científico Médicas Españolas (FACME), la Conferencia Nacional de Decanos de Facultades de Medicina (CNDFM), el Consejo Nacional de Especialidades en Ciencias de la Salud (CNECS) y el Consejo Estatal de Estudiantes de Medicina (CEEM) exponen, a través de este documento, las razones de esta defensa que tiene como objetivo la calidad de la asistencia sanitaria, en línea con el acuerdo firmado en 2013 con el Ministerio de Sanidad, Servicios Sociales e Igualdad para la sostenibilidad del SNS.

\section{Análisis de la situación actual}

Algo en lo que todos los españoles estamos de acuerdo, y de lo que incluso nos sentimos orgullosos, es que disfrutamos de una buena asistencia sanitaria a la que no queremos renunciar. Sin duda, el buen funcionamiento del SNS se asienta en la calidad de la formación de los profesionales sanitarios, siendo los médicos uno de sus pilares fundamentales. Por lo tanto, coincidiremos en que garantizar la formación de los médicos es vital y que es una tarea que hay que tomarse muy en serio. En caso contrario, un buen programa de gestión sanitaria no daría los frutos deseados y la financiación requerida se multiplicaría al necesitarse un mayor gasto para atender las mismas necesidades asistenciales.

La formación del médico es larga, con una duración mínima de 11 años, y sacrificada. Comienza en la facultad de medicina, a la cual sólo acceden los alumnos más brillantes de bachillerato, quienes tras un difícil y tortuoso procedimiento para conseguir matricularse, que es necesario modificar, logran en seis años el título de Grado en Medicina. Posteriormente deben superar una prueba selectiva, y a la
Foro de la Profesión Médica de España: Organización Médica Colegial de España (OMC), Federación de Asociaciones Científico Médicas Españolas (FACME), Confederación Estatal de Sindicatos Médicos (CESM), Conferencia Nacional de Decanos de Facultades de Medicina (CNDFM), Consejo Nacional de Especialidades en Ciencias de la Salud (CNECS), Consejo Estatal de Estudiantes de Medicina (CEEM).

(c) 2014 FEM 
vez distributiva, para acceder a la etapa de formación especializada (MIR, 4-5 años); entre ambas etapas dedican un año adicional para la preparación del examen y acceder a la plaza de MIR. Hay que tener en cuenta que el joven que ha logrado el Grado en Medicina ha obtenido el título de médico, pero en realidad sólo nominalmente, pues no está habilitado para trabajar en el SNS y su acceso al sector asistencial privado es prácticamente imposible. De hecho, los graduados en medicina sólo se convierten en médicos con capacidad para el ejercicio asistencial autónomo tras finalizar su periodo MIR, por lo que las dos etapas de la formación médica se deben concebir de forma unitaria. Tal circunstancia establece una diferencia entre los recién graduados en medicina con el resto de los titulados sanitarios, que aunque tampoco tienen fácil acceder al mercado de trabajo, no requieren necesariamente un periodo de especialización para ejercer en el SNS (enfermeros, fisioterapeutas...) o en el sector privado (enfermeros, odontólogos, farmacéuticos, fisioterapeutas...).

¿Qué consecuencias tiene que sea necesario el periodo MIR para ejercer labores asistenciales? La respuesta es sencilla: como existe un numerus clausus para acceder al MIR, dicha oferta debe estar equilibrada con el número de egresados de nuestras facultades. En caso de existir desfases entre ellas, no se cubrirían las plazas de MIR ofertadas o los recién graduados, médicos nominales, no podrían finalizar su proceso de formación y entrarían a formar parte de una bolsa de titulados sin posibilidades de ejercicio asistencial. En este último caso, los jóvenes egresados habrían perdido su tiempo y esfuerzo; recordemos el lema de la protesta estudiantil de hace unos años: $6=0$. A este coste personal hay que sumarle el económico y social: pensemos en el consiguiente dispendio económico que suponen los recursos desaprovechados en seis años de formación y que la bolsa de médicos nominales estaría constituida por egresados brillantes que hubieran podido convertirse en excelentes profesionales en otros ámbitos muy necesarios para el desarrollo del país.

Para evitar estas situaciones es necesario que el numerus clausus se aplique al comienzo del proceso formativo, es decir, en el acceso a los estudios de grado en la facultad de medicina. Este numerus clausus debe calcularse correctamente, para lo cual es imprescindible una planificación adecuada de las necesidades de facultativos especialistas -responsabilidad de las autoridades sanitarias- que determine la oferta de plazas de MIR. Por supuesto, hay que tener en cuenta otros factores como los desfases temporales y la tasa de abandono durante el gra- do para definir el número de estudiantes que deben ingresar en las facultades de medicina.

Las razones expuestas anteriormente justifican per se la existencia del numerus clausus para estudiar medicina. Pero existe una segunda razón, que no es menos importante: debemos garantizar la calidad de la formación de la primera etapa del médico en nuestras facultades. La etapa universitaria es fundamental para la formación del médico y su calidad está condicionada, además de por la coherencia de los planes de estudios e instalaciones apropiadas, por un profesorado con la formación adecuada y la capacidad docente del sistema hospitalario. En efecto, el profesorado necesita una formación específica y no todos nuestros hospitales se pueden utilizar para la docencia de pregrado (éstos deben superar los criterios publicados en el BOEA-1987-18294 para su acreditación). En consecuencia, profesorado apropiado y hospitales acreditados limitan el número de estudiantes que pueden atender. Es importante aclarar que nuestros vecinos europeos también aplican el numerus clausus al comienzo de la etapa formativa del médico, basándose en criterios similares.

Una vez expuestas las razones que justifican el numerus clausus para acceder a las facultades de medicina, debemos analizar:

- El escenario actual de la formación de grado en medicina.

- Las instituciones que tienen la responsabilidad de determinar el número de estudiantes y de facultades de medicina que España necesita para atender a la oferta MIR.

Para comprender el escenario actual de la formación en medicina es conveniente cierta perspectiva histórica. El numerus clausus comenzó a aplicarse en nuestras facultades de medicina en 1994 como respuesta a la masificación que afectaba a la calidad de la formación y al 'paro' que padecía la profesión. Parar fijar su número se tuvieron en cuenta las recomendaciones de la Organización Mundial de la Salud (1 estudiante por 10.000 habitantes) y algunas de las consideraciones expuestas anteriormente. Así, en el año 2002, tras un periodo de ajustes, llegamos a los 4.350 estudiantes de nuevo acceso que se repartían en 27 facultades (26 públicas y una privada). En esos años, el número total de médicos en España parecía razonable para nuestro sistema sanitario, aunque sólo se disponía de un censo estimativo de facultativos. La profesión médica parecía encontrarse en equilibrio, no existía paro médico y el estudiante que accedía a nuestras facultades de medicina tenía asegurada una plaza de formación 
especializada que le habilitaba para ejercer en el sistema sanitario. Ante esas expectativas, los estudiantes más brillantes que terminaban el bachillerato decidían estudiar medicina.

En los años siguientes, el entorno de prosperidad nacional determinó que España se convirtiera en destino para la inmigración aumentando la población, especialmente la infantil. Se inauguraron hospitales y centros de salud, sin una planificación adecuada, que colaboraron a incrementar la demanda de facultativos de determinadas especialidades. Comenzaba a oírse el mensaje confuso de que en España se necesitaban más médicos y que el déficit sería todavía mayor en el futuro. En realidad, sólo eran necesarios facultativos en determinadas especialidades y en algunos casos causados por una mala distribución geográfica.

Ante esta situación, nuestros gobernantes, que aún seguían con un censo de médicos sólo estimativo, realizaron una planificación de necesidades de médicos que después se ha demostrado no acertada (según los expertos, no se puede hablar sobre necesidades sin plantearse, de forma paralela, para qué sistema de salud o para qué modelo asistencial). Las autoridades sanitarias incrementaron de forma considerable, sin priorizar necesidades de especialistas, la oferta de plazas de MIR, de forma que se produjo un desequilibrio importante entre dicha oferta y los licenciados egresados de nuestras facultades. Para solventar estos déficits llegaron a nuestro país un importante número de médicos que convalidaron sus títulos directamente en el Ministerio de Educación o el de especialistas en el Ministerio de Sanidad, algunos de ellos sin las necesarias garantías. Entre 2001 y 2011 se convalidaron más títulos extracomunitarios (50.205) que médicos se han licenciado en las facultades españolas (46.194). Sólo en el año 2010 se reconocieron 271 títulos europeos y se homologaron 5.928 títulos extracomunitarios por el Ministerio de Educación y 298 por las comisiones nacionales de distintas especialidades. De hecho, en la convocatoria MIR de 2009, de las 6.948 plazas que se ocuparon, 2.369 lo fueron por licenciados extracomunitarios, aunque posteriormente se han aplicado restricciones (en la convocatoria actual son el $4 \%$ de las plazas).

El confuso mensaje de la necesidad de médicos que responsables políticos e incluso alguna asociación y colegio profesional difundían, produjo un enorme 'efecto llamada' para ingresar en nuestras facultades ante las buenas perspectivas de empleo. La propia sociedad presionaba a la universidad para que se incrementaran el número de plazas en las facultades ante la enorme demanda de estudiantes, y se comenzaron a promover facultades de medicina tanto públicas como privadas. Ante el estupor de algunos, entre los que se situaba la CNDFM, de 2005 a 2012 pasamos de 4.350 a más 7.000 alumnos de nuevo ingreso. Toda ciudad de cierta entidad quería tener una facultad de medicina, y las de mayor población, aumentar su número: pasamos de 28 a 40 facultades (cinco públicas y siete privadas nuevas; en 2003 se había creado la facultad de la Universidad CEU San Pablo) y nos convertimos en el segundo país de población superior a 20 millones de habitantes con más facultades de medicina por habitante. Pero, como supondrán, los resultados de incrementar el número de estudiantes y facultades tardan un tiempo: hay que esperar seis años para que un estudiante se pueda presentar al examen MIR y al menos cuatro años para que sea especialista. Es ahora cuando esas políticas de incrementos incontrolados empiezan a evidenciarse.

Así hemos llegado a la situación actual en la que, resultado de esta torpe y desafortunada planificación, en noviembre de 2013, 2.389 médicos están en 'paro', 4.203 son demandantes de empleo y comenzamos a exportar médicos especialistas (3.400 en 2013). Este año y en los próximos se sumarán nuevas generaciones que terminan su periodo MIR y competirán en el escaso mercado de trabajo. Para agravar más este escenario, debido a la situación económica actual y dentro de una reciente planificación sanitaria que trata de disminuir el gasto y mejorar la eficiencia del SNS, la oferta de plazas de MIR ha empezado a disminuir (6,7\% en 2012 y $3,4 \%$ en 2013). En consecuencia, si no reducimos el $n u$ merus clausus en nuestras facultades, sufriremos importantes desajustes entre los graduados y las plazas de MIR ofertadas. A lo anterior se añade que un número importante de jóvenes especialistas que no encuentran empleo o algunos que deciden cambiar de especialidad se vuelven a presentar al examen MIR junto a los recién egresados y extracomunitarios. Por tanto es previsible que, de continuar esta tendencia, se produzca una bolsa de médicos 'nominales' que superará ampliamente los 1.000 anuales y que, al no poder convertirse en especialistas, no podrán alcanzar el ejercicio de la profesión. Teniendo en cuenta estas y otras consideraciones, los expertos en demografía médica estiman que es necesario disminuir el número de nuevos alumnos a 5.500-6.000 para equilibrar las plazas de MIR que presumiblemente se convocarán dentro de seis años para atender, a su vez, las necesidades de médicos especialistas dentro de 11 años.

En este contexto, mientras algunas universidades públicas han comenzado a disminuir su núme- 
ro de estudiantes, sin embargo otras anuncian su incremento. Por si fuera poco, y para complicar más este escenario, algunas comunidades autónomas han autorizado -o están en vías de autorizar- nuevas universidades privadas que tienen prevista la titulación de medicina (Mare Nostrum University y Universidad Católica de Alicante) y algunas universidades públicas no abandonan definitivamente la idea de abrir nuevas facultades (Universitat de les Illes Balears, Universidad de Jaén, Universidad de Almería y Universidad de Huelva) y otras privadas (Universitat de Vic) anuncian sus planes de abrir una facultad de medicina.

\section{Responsables de regular el número de estudiantes de grado de medicina y de autorizar nuevas facultades de medicina}

Respecto al numerus clausus, el órgano responsable es la Conferencia Nacional de Política Universitaria (órgano formado por el Ministerio de Educación y las consejerías de educación). En ella se decide el número total de alumnos admitidos y su distribución teniendo en cuenta las estimaciones del Ministerio de Sanidad y las propuestas de las consejerías autonómicas, a su vez originadas en las facultades de medicina y aprobadas por las respectivas universidades.

La puesta en marcha de una nueva facultad es un proceso complicado. Para que una universidad pueda impartir un titulo de grado, es decir, para crear una nueva facultad de medicina, debe contar con la autorización de su respectiva consejería de educación y que su plan de estudios sea verificado por el Consejo de Universidades -órgano formado por el Ministerio de Educación y por la Conferencia de Rectores de Universidades Españolas (CRUE) - tras el preceptivo informe favorable de la Agencia $\mathrm{Na}$ cional de Evaluación de la Calidad y Acreditación (ANECA) o las agencias autonómicas acreditadas. Dichas agencias valoran si el plan es coherente y acorde con las directrices del título, y en función de los recursos humanos (plantilla de profesores) y de las instalaciones disponibles (se incluyen los hospitales concertados públicos o privados para las prácticas clínicas) podrá emitir un informe favorable para impartir el titulo de Grado en Medicina limitado a un determinado número máximo de alumnos.

En resumen:

- El numerus clausus de alumnos de nuevo ingreso y su distribución entre las facultades de medi- cina se establece por la Conferencia Nacional de Política Universitaria (Ministerio de Educación y consejerías de educación).

- La aprobación de los planes de estudios de grado en medicina depende del Consejo de Universidades (Ministerio de Educación y CRUE) tras el preceptivo informe favorable de la ANECA, o agencias regionales acreditadas, y la autorización para su implantación corresponde a las comunidades autónomas.

Tras este análisis nos preguntamos: ¿cómo es posible que actualmente la Conferencia Nacional de Política Universitaria comience a reducir, aunque tímidamente, las plazas para estudiar el grado en medicina (1,35\%), mientras que el Consejo de Universidades y las comunidades autónomas aprueban la creación y puesta en marcha de nuevas facultades de medicina?

Está claro que no es congruente reducir el número de estudiantes y, al mismo tiempo, abrir nuevas facultades de medicina.

Es fácil darse cuenta de que el escenario es complejo y que en él se mueven de manera descoordinada varios agentes con distintos intereses: universidades privadas (promovidas por grupos empresariales, fundaciones e instituciones religiosas), universidades públicas, consejerías de educación y de sanidad de las comunidades autónomas, Ministerio de Educación y Ministerio de Sanidad, ANECA y agencias autonómicas acreditadas.

\section{Propuestas}

El Foro de la Profesión Médica de España, que agrupa a la OMC, la CESM, la FACME, la CNDFM, el CNECS y el CEEM, y que recientemente ha firmado un acuerdo con el Ministerio de Sanidad para la sostenibilidad del SNS, se ve en la obligación de hacer públicos nuestros análisis y reflexiones ante la situación descrita y exponer las siguientes propuestas:

- Mayor coordinación entre las instituciones representativas de la profesión y las autoridades sanitarias y educativas, para garantizar una formación de calidad en nuestras facultades que resuelva las necesidades futuras de médicos.

- Realizar una planificación que adecue el numerus clausus a estas necesidades reales de las plazas de MIR en los hospitales acreditados.

- Que se frene el crecimiento del número de facultades de medicina. 